\title{
Quasi-One-Dimensional Photonic Crystal as a Compact Building-Block for Refractometric Optical Sensors
}

\author{
Wico C. L. Hopman, Student Member, IEEE, Pierre Pottier, Didit Yudistira, Joris van Lith, Paul V. Lambeck, \\ Richard M. De La Rue, Fellow, IEEE, Alfred Driessen, Senior Member, IEEE, Hugo J. W. M. Hoekstra, and \\ René M. de Ridder, Member, IEEE
}

\begin{abstract}
We report the fabrication and the characterization of the refractometric and thermo-optical properties of a quasi-one-dimensional waveguide photonic crystal-a strong, 76- $\mu \mathrm{m}$-long Bragg grating. The transmission spectra (around 660 $\mathrm{nm}$ ) of the structure have been measured as a function of both the cladding refractive index and the temperature. The transmission stopband was found to shift by $0.8-\mathrm{nm}$ wavelength for either a cladding refractive index change of 0.05 or a temperature change of $120 \mathrm{~K}$. The steep stopband edges provide a sensitive detection method for this band shift, by monitoring the transmitted output power.
\end{abstract}

Index Terms-Bragg, grating, photonic crystal, sensor, silicon nitride, waveguide.

\section{INTRODUCTION}

A STRUCTURE having a periodic index modulation in one dimension is known as a one-dimensional (1-D) photonic crystal. If the structure has nonperiodic features in the other two dimensions, it is denoted as a quasi-1-D photonic crystal. We consider a wide ridge-type channel waveguide of silicon nitride with a grating etched into the core layer.

Besides their use in theoretical studies for computationally efficient modeling of selected properties of quasi-two-dimensional (2-D) photonic crystals (also known as photonic crystal slabs), e.g. [1] and [2], such grating structures have found direct application as distributed Bragg reflectors and the like in semiconductor lasers, and fiber Bragg gratings, especially for optical wavelength filtering and dispersion compensation in optical communication networks [3], [4]. They have also been used as sensing structures, both in fiber devices [5] and in planar integrated optics [6]. In many of these Bragg grating applications, one is interested in obtaining a narrow reflection peak and low transmission losses outside the peak in order to obtain a selective and efficient optical wavelength filter. Such characteristics require a relatively long grating with a weak index modulation.

Manuscript received July 15, 2004; revised October 29, 2004. This work was supported by the Mesa+ Research Institute and by the University of Glasgow.

W. Hopman, D. Yudistira, J. van Lith, P. V. Lambeck, A. Driessen, H. J. W. M. Hoekstra, and R. M. de Ridder are with the Integrated Optical MicroSystems Group, MESA+ Research Institute, University of Twente, 7500 AE Enschede, The Netherlands (e-mail: W.C.L.Hopman@ewi.utwente.nl).

P. Pottier and R. M. De La Rue are with the Optoelectronics Research Group, Department of Electronics and Electrical Engineering, University of Glasgow, G12 8QQ Glasgow, Scotland, U.K. (e-mail: Pottier@elec.gla.ac.uk).

Digital Object Identifier 10.1109/JSTQE.2004.841693
A Bragg grating with a strong index modulation shows a typical property of photonic crystals: an extended transmission stopband. Although such a structure does not possess a full photonic bandgap (i.e., overlapping stopbands for all propagation directions and polarizations), it can still have a useful transmission stopband, since the channel waveguide strongly limits the angular spectrum of incident waves. The width of this stopband and the steepness of its edges increase with the strength of the refractive index modulation.

For optical sensing purposes, we propose to exploit the steep edges of the stopband of a strong grating. The central wavelength and the edges of the stopband shift toward longer wavelengths with increasing average index of the structure. A small wavelength shift of such an edge can cause a large change in the transmitted power from a source having an appropriate wavelength. The Bloch modes of a strong grating can have a large overlap with the cladding material, providing an efficient mechanism for detecting index changes of, e.g., a fluid cladding. Although the losses at the long wavelength stopband edge can reach low levels [1], strong gratings are often considered less suitable for practical applications due to out-of-plane diffraction loss. In fact, this may be of key importance considering telecom devices, contrary to most sensor applications, where this property can well be the measuring principle [7] For example, it is sometimes immaterial whether a transmission minimum is caused by back reflection or out-of-plane scattering.

A key advantage of the proposed device is that the sensing elements can be very compact (in this work, $76 \mu \mathrm{m}$ long, compared to typically $4 \mathrm{~cm}$ for a Mach-Zehnder interferometer, as in [8], or a few millimeters for surface plasmon resonance sensors, e.g., [9]), opening the possibility of integrating an array of sensors, each for detecting a different measurand or covering a different range (e.g., of concentrations). The small measurand volume needed and the resolution obtained are similar to those of a recently demonstrated micro ring resonator based sensor [10].

It has been demonstrated that the bandgap of a photonic crystal that is infiltrated with a liquid crystal can be widely tuned due to the thermally induced refractive index change of the liquid crystal [11]. In addition, thermal tuning of resonance frequencies of cavities in semiconductor photonic crystals has been shown [12]. We show that the much smaller thermooptic effect of $\mathrm{Si}_{3} \mathrm{~N}_{4}$ and $\mathrm{SiO}_{2}$ can be used for tuning the stopband over a small range, which is, however, easily detectable by 


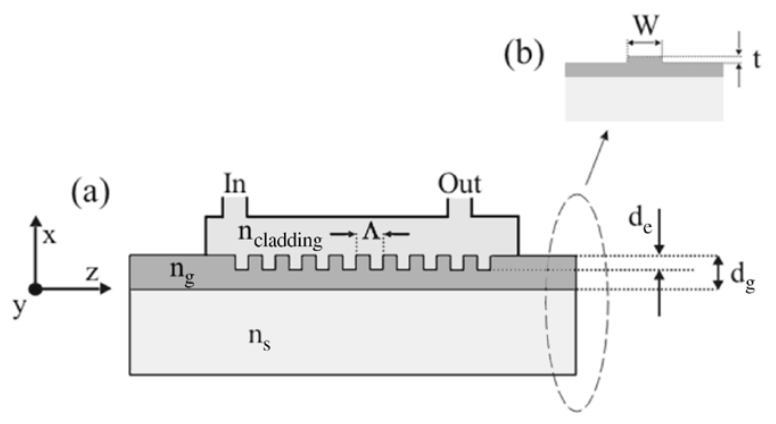

Fig. 1. (a) Cross section of the refractive-index sensor. The sensing element is a quasi-1-D photonic crystal, a strong grating in a $\mathrm{Si}_{3} \mathrm{~N}_{4}$ shallow ridge-type waveguide. The top cladding over the grating is formed by a fluid (the measurand) contained in a cuvette that is sealed to the sensor chip. $d_{e}=22 \mathrm{~nm}, d_{g}=212 \mathrm{~nm}, n_{g}=2.01, n_{s}=1.46$, the value of $n_{\text {cladding }}$ is varied, $\Lambda=190 \mathrm{~nm}$. (b) A schematic side view of the ridge waveguide, $t=2 \mathrm{~nm}, W=2 \mu \mathrm{m}$.

observing the optical transmission near a band edge. This effect may be used for tuning the structure into its optimum operating point (with respect to the source wavelength) or for optical switching. Although we used bulk heating of the entire structure for measuring the thermooptic actuation, like [12], it has recently been demonstrated that in spite of their strongly nonplanar surface, photonic crystal structures can be thermally tuned using small local heaters [13], [14].

\section{EXPERIMENT}

\section{A. Device Fabrication}

A schematic cross section of the investigated device, which is based on the structure that was modeled in [1], is shown in Fig. 1. The optical part of the structure is a three-layer waveguiding-system with a 212-nm-thick $\mathrm{Si}_{3} \mathrm{~N}_{4}$ guiding layer $\left(n_{g}=\right.$ $2.01)$, grown on top of a $9-\mu$ m-thick $\mathrm{SiO}_{2}$ buffer layer $\left(n_{s}=\right.$ $1.46)$ on silicon. The top cladding (refractive index $n_{\text {cladding) }}$ ) is either air, or a fluid of which the index could be varied. A shallow, 2- $\mu \mathrm{m}$-wide ridge with a 2-nm step height, forming a single-mode waveguide at $\lambda>450 \mathrm{~nm}$, was etched into the $\mathrm{Si}_{3} \mathrm{~N}_{4}$ layer using conventional photolithography and wet chemical etching. The grating period was chosen to be $\Lambda=190 \mathrm{~nm}$, leading to a stopband located in the red visible spectrum. It has 401 periods, resulting in an overall length of $76.19 \mu \mathrm{m}$. The width of the grating is $80 \mu \mathrm{m}$ to ensure full overlap with the evanescent field tails in the slab region next to the ridge. The grating was defined with its grooves perpendicular to the axis of the ridge waveguide using direct e-beam writing, and etched into the $\mathrm{Si}_{3} \mathrm{~N}_{4}$ guiding layer using an intermediate Titanium mask and reactive ion etching. After etching, an atomic force microscope (AFM) was used for measuring the filling factor (groove width $60 \%$ of $\Lambda$ ) and the etch depth (22 nm, i.e. approximately $10 \%$ of the guiding layer thickness). A small cuvette was placed over the photonic crystal area, and sealed with silicone paste, so that the top cladding of the structure could be replaced by a fluid.

\section{B. Measurement Setup}

The sensor is characterized by measuring transmission spectra as a function of a measurand. This is carried out by

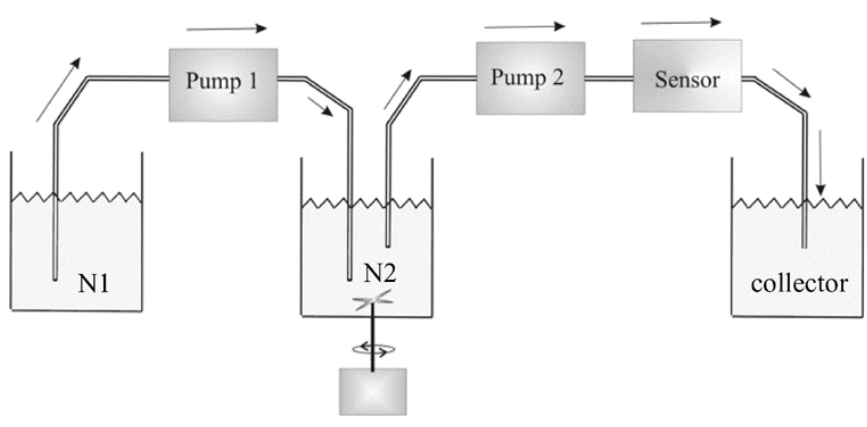

Fig. 2. Setup for characterizing the refractometer. At the start of a measurement, vessels $\mathrm{N} 1$ and $\mathrm{N} 2$ contain different, mixable fluids. Pump 1 slowly adds fluid from vessel N1 to the stirred vessel N2, producing a mixture of which the change of refractive index with time can be calibrated. Pump 2, having the same flow rate as pump 1 , feeds the mixture through the cuvette, so that the refractive-index sensitivity of the sensor can be determined by measuring the light transmission versus time.

using a tunable dye laser [620-700 nm] and a simple photodetector. In all cases, TE-polarized light was used, which was coupled into the structure using the commonly used end-fire setup, consisting of a half-wave plate, polarizer, and two $\times 40$ microscope objectives for in and output coupling.

1) Refractive Index Measurements: The sensitivity of the sensor for changes in the cladding index could be measured by feeding different fluids with known refractive indexes through the cuvette, using the setup shown schematically in Fig. 2. A continuous change in refractive index could be realized by slowly mixing together two fluids of different refractive indexes. At the start of the measurement, the closed vessel N2 contained pure ethanol (refractive index $n=1.36$ ). Vessel $\mathrm{N} 1$ contained a benzyl-alcohol with a refractive index of 1.54 , which is $13 \%$ higher than that of ethanol. Pump 1 slowly added benzyl alcohol to the fluid in vessel N2 which was stirred; both fluids are well mixable. After feeding the mixture through a relatively small volume cuvette the fluid was collected in a third vessel. During the measurements, calibration data were collected by monitoring the refractive index in vessel N2 using an Abbe refractometer at a wavelength of $632.8 \mathrm{~nm}$. The flow system illustrated in Fig. 2 is characterized by a set of simple differential equations. Using the calibration data, the parameters that were originally known with insufficient accuracy, could be determined using a fitting procedure. After calibration, the index at each moment in time was known with an accuracy of approximately $5 \times 10^{-4}$.

2) Temperature Measurements: The temperature of the sensor was adjusted by heating the entire chip. The temperature was measured using a thermocouple. Due to the bulk heating procedure, the optical alignment of the setup tended to drift after adjusting the temperature. A period of approximately 30 min after an adjustment had been made, was needed for stabilization.

\section{MeAsurement Results}

The transmission spectra that were measured at the start and at the end of a refractive index scan using the setup of Fig. 2 are presented in Fig. 3. The first spectrum was measured with pure ethanol $(n=1.36)$, the second one with a mixture $(n=1.528)$ 


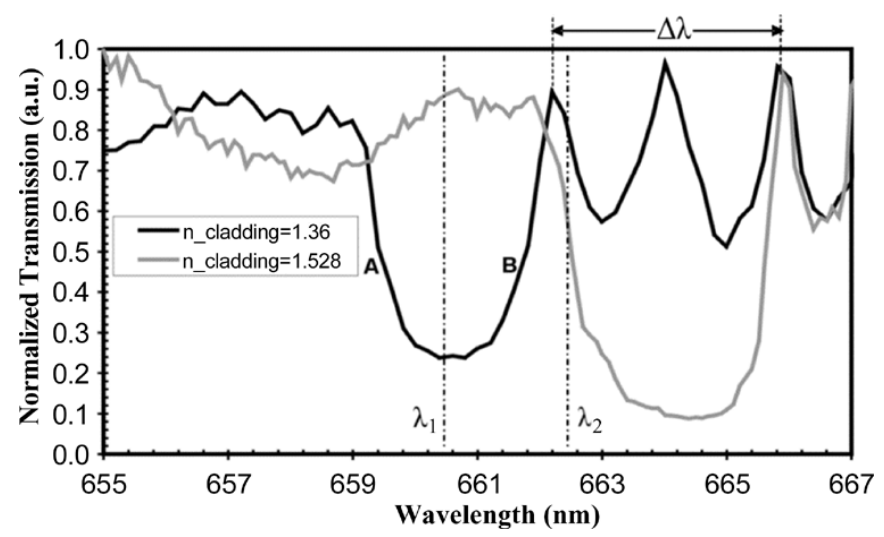

Fig. 3. Normalized transmission spectra for two different cladding indices $\left(n_{\text {-cladding }}\right)$. The air band edge and the dielectric band edge are labeled with the letters A and B, respectively. Wavelengths $\lambda_{1}$ and $\lambda_{2}$ are fixed for probing edges $\mathrm{A}$ and $\mathrm{B}$, respectively, leading to the characteristics shown in Fig. 4.

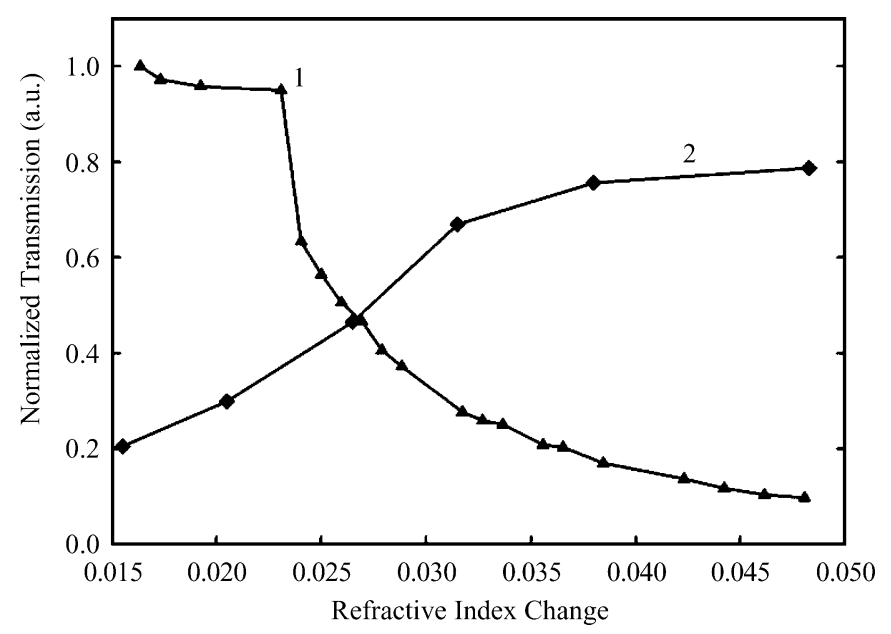

Fig. 4. Normalized transmission versus the change in cladding index. Curve 1 (scanning edge $\mathrm{A}$ ) is measured at $\lambda_{1}$ and curve 2 (edge $\mathrm{B}$ ) is measured at $\lambda_{2}$ (c.f. Fig. 3). Both curves are normalized to the maximum transmitted power, for curve 1 and 2, respectively, 360 and $635 \mathrm{nW}$.

of ethanol and benzyl-alcohol. A wavelength shift (shift of edge $B$ ) of about $4 \mathrm{~nm}$ was found for a cladding index change of 0.168 .

The following variation of the measurement method exploits the steep edges of the transmission stopband. By choosing a measurement wavelength to be approximately halfway the slope of the band edge (the wavelengths $\lambda_{1}$ and $\lambda_{2}$ are chosen outside the slope region so that the edges A and B, respectively, could be characterized completely, see Fig. 3), a change in the cladding index can be very accurately determined by monitoring the transmitted power. A small change in cladding index will shift the stopband to higher or lower wavelength, depending on the sign of the index change. This shift causes a corresponding large change in output power due to the steep slope of the band edge. Setting $\lambda_{1}=660.5$ and $\lambda_{2}=662.5 \mathrm{~nm}$, the results shown in Fig. 4 were obtained. The figure shows that the maximum sensitivity is found at an index change of 0.024 for curve 2 . In order to arrive at a numerical value for obtained sensitivity, we use, however, a conservative estimate based on the more gradual slope of curve 1 .

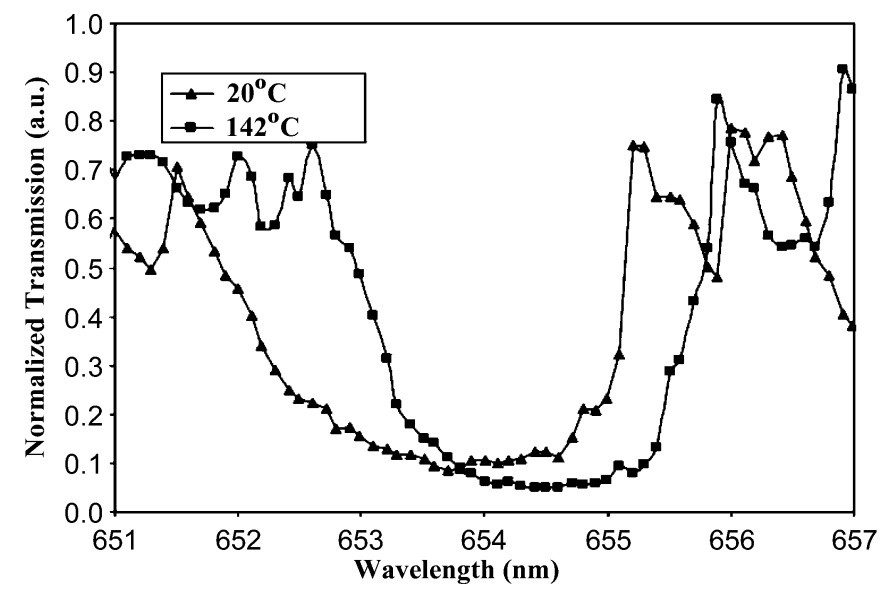

Fig. 5. Normalized transmission spectra for two different temperatures.

It is clear that both band edges show a marked output power change with a change of cladding index. We define the sensitivity $S$ of the sensor as

$$
S=\frac{1}{P} \frac{\partial P}{\partial n}
$$

The values of the transmitted power $P$ and its derivative with respect to the cladding refractive index $\partial P / \partial n$ can be determined from Fig. 4, leading to a conservative estimate of $S \cong 24$ (based on the average slope of curve 1).

With the simple photodetector setup we used, a resolution $\eta$ of $1 \%$ of the maximum transmitted power could be obtained. The minimum detectable cladding index change $\Delta n$ is

$$
\Delta n=\frac{\eta}{S}
$$

for this sensor evaluating to a value $\Delta n \cong 4 \times 10^{-4}$. Approximately the same value can be found directly from Fig. 3 by estimating $S$ from the spectral shift of the stopband for the given index change, and the slope of the band edge.

The thermal tunability of the photonic crystal is demonstrated by the normalized transmission spectra for two different temperatures shown in Fig. 5. A 0.8-nm shift of the center stopband wavelength is observed for a $122 \mathrm{~K}$ temperature change, corresponding to a thermal tuning coefficient $\partial \lambda / \partial T \cong 7 \pm 1 \mathrm{pm} / \mathrm{K}$. As mentioned in Section II-B2), the measurement setup suffered from drift of the in-coupling conditions. Since the measurement of a single spectrum took several minutes, the distortion of the transmission spectrum may be partly attributed to this effect.

Fig. 6 shows the center wavelength versus temperature. Between measurement points, we allowed the setup to stabilize for at least $30 \mathrm{~min}$. As should be expected, a linear relation was found. The average thermal tuning coefficient found from this more accurate measurement was $\partial \lambda / \partial T \cong 6.7 \mathrm{pm} / \mathrm{K}$.

A calculation with a 2-D bidirectional eigenmode propagation method using the known thermo-optic coefficients of $\mathrm{Si}_{3} \mathrm{~N}_{4}$ and $\mathrm{SiO}_{2}$ (both approximately $1 \times 10^{-5}$ ), and the geometric parameters of the structure, results in a value of $\partial \lambda / \partial T=4 \mathrm{pm} / \mathrm{K}$. The discrepancy may be attributed to the effect of thermal expansion of the bulk silicon substrate and the elastooptic effect, which the model did not account for. 


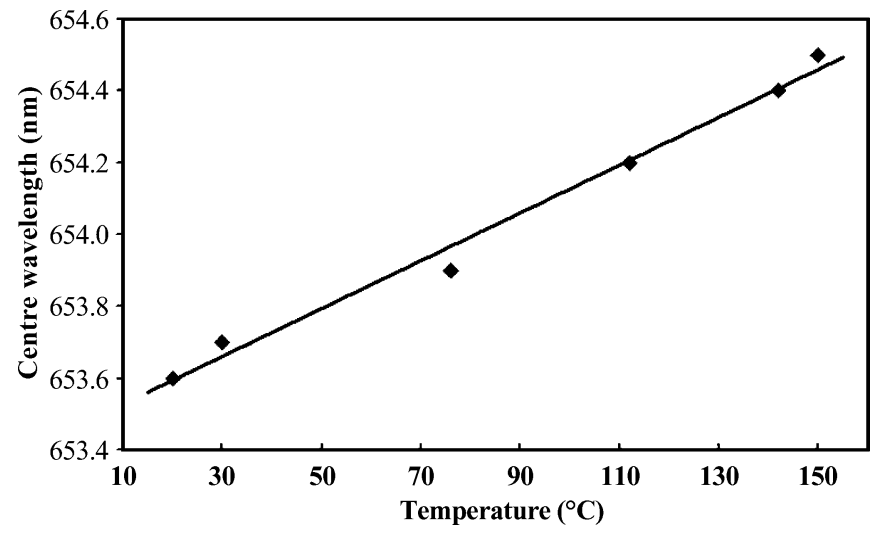

Fig. 6. Center wavelength (i.e., the wavelength exactly in the middle of the stopband defined by the $-3-\mathrm{dB}$ point of the stopband edges) versus temperature.

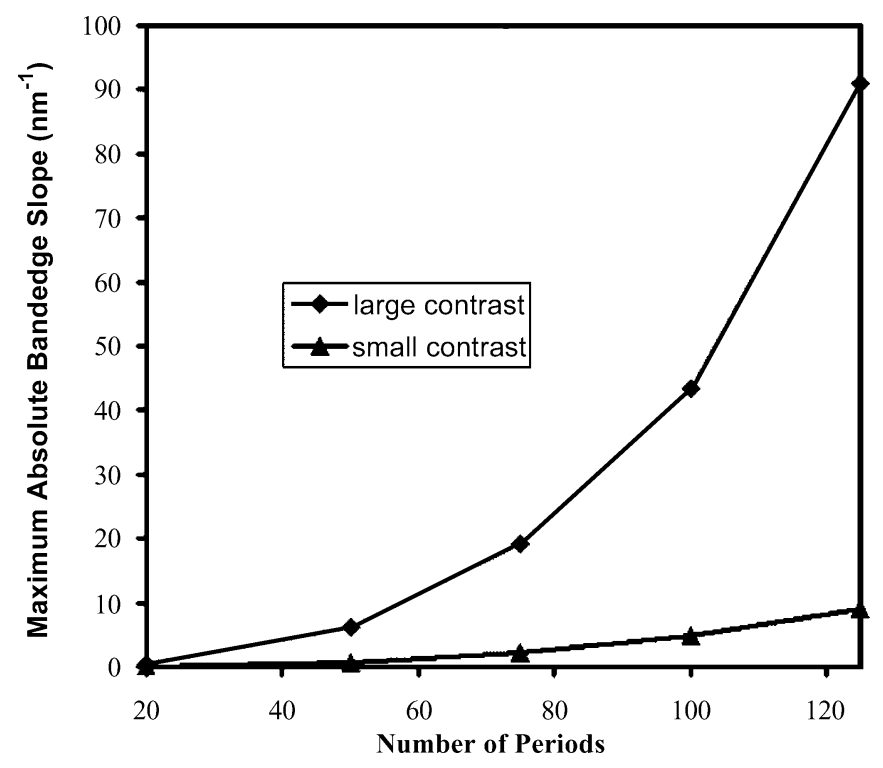

Fig. 7. Maximum slope of the band edge $\partial P / \partial \lambda$ versus the number of grating periods. Increasing the contrast in this 1-D model corresponds to increasing the etching depth of the grating. The "small contrast" approximately represents the fabricated structure; a contrast of 0.2. An refractive index contrast of 0.8 was taken for the "large contrast."

\section{DISCUSSION}

The photonic crystal structure used in the measurements was not optimized for sensing purposes, therefore, a further detailed investigation is needed to determine the maximum achievable improvement of the sensitivity. As expected, a wavelength shift of the photonic band edges was observed on changing the cladding index. The results prove that this photonic crystal device can be used as a sensitive integrated device for determining the cladding index. Expanding (1), we obtain

$$
S=\frac{1}{P} \frac{\partial P}{\partial n}=\frac{1}{P} \frac{\partial P}{\partial \lambda} \frac{\partial \lambda}{\partial n}
$$

clearly showing that the sensitivity can be enhanced by increasing the slope of the band edge, $\partial P / \partial \lambda$. One way to do this is by increasing the number of periods of the grating. Fig. 7 shows the results from a 1-D photonic crystal model for the maximum slope (at the band edge) versus the number of periods (length of the grating). The model is based on an effective index approximation and a plane wave expansion. Fig. 7 indicates that the sensitivity of this device is increasing stronger than linear with the number of periods in the computed range. Since, in the actual three-dimensional device, loss increases with increasing number of periods, an optimum number of periods may exist. Further study and more computational resources are needed to validate this behavior outside the computed region. From Fig. 7, we can conclude that increasing the etch depth and thus increasing the contrast will also strongly enhance the slope $\partial P / \partial \lambda$.

The optical insertion loss of the device can be significantly reduced by using an optimized fiber-chip coupling (e.g., [8]). Clever signal processing, e.g., by simultaneously using both band edges in a push-pull way (requiring two light sources at different wavelengths) will increase the sensitivity. The resolution $\eta$ can be increased by a factor of 10 , from $1 \%$ to $0.1 \%$, by using a more sophisticated photodetection setup.

The resulting increase in sensitivity of at least two orders of magnitude will make this photonic crystal sensor an interesting building-block for densely integrated optic sensor arrays. The stability problems and slow response time observed with bulk thermal actuation can both be considerably reduced by using a local heater as in [13], [14]. Using an integrated fiber-chip coupling will also be very beneficial for increasing stability.

\section{CONCLUSION}

We have demonstrated a very compact refractometer sensor based on a short deeply etched grating. An increase of the cladding refractive index causes a shift of the transmission spectrum of the grating toward longer wavelengths of approximately $23 \mathrm{~nm}$ per unit index change. The steep slopes of the photonic band edges (in our case especially, the dielectric band edge at the long-wavelength side of the gap) can be used for increasing the sensitivity of the device. With a simple photodetector, a variation of $4 \times 10^{-4}$ in the cladding index could be detected. A thermally induced spectral shift of approximately 7 $\mathrm{pm} / \mathrm{K}$ was observed.

Besides an improvement of resolution by two orders of magnitude by changing the detection equipment alone, we believe that also the optical design of devices based on this sensing principle can be further optimized. Although Mach-Zehnder interferometers will probably remain the devices of choice if the ultimate in sensitivity is to be attained, the deep grating device presented in this paper may offer a good compromise between size and sensitivity that is requiregd for the present and future large scale integrated sensor arrays.

\section{ACKNOWLEDGMENT}

The authors would like to thank E. Klein for fruitful discussions and for help with the temperature characterization.

\section{REFERENCES}

[1] J. Čtyroký et al., "Bragg waveguide grating as a 1-D photonic band gap structure: Cost 268 modeling task," Opt. Quantum Electron., vol. 34, pp. 455-470, 2002.

[2] W. Bogaerts, P. Bienstman, D. Taillaert, R. Baets, and D. de Zutter, "Out-of-plane scattering in 1-D photonic crystal slabs," Opt. Quantum Electron., vol. 34, pp. 195-203, 2002. 
[3] D. Gauden, E. Goyat, and A. Mugnier et al., "A tunable four-channel fiber Bragg grating dispersion compensator," IEEE Photon. Technol. Lett., vol. 15, no. 10, pp. 1387-1388, Oct. 2003.

[4] L. G. Sheu, K. P. Chuang, and Y. C. Lai, "Fiber Bragg grating dispersion compensator by single-period overlap-step-scan exposure," IEEE Photon. Technol. Lett, vol. 15, no. 7, pp. 939-941, Jul. 2003.

[5] W. Jin, Y. Zhou, P. K. C. Chan, and H. G. Xu, "A fiber-optic grating sensor for the study of flow-induced vibrations," Sens. Actuators A, Phys., vol. 79, pp. 36-45, 2000.

[6] G. J. Veldhuis, J. H. Berends, R. G. Heideman, and P. V. Lambeck, "An integrated optical Bragg-reflector used as a chemo-optical sensor," Pure Appl. Opt., vol. 7, pp. L23-L26, 1998.

[7] J. van Lith, P. V. Lambeck, H. J. W. M. Hoekstra, and R. G. Heideman, "The segmented waveguide sensor: Principle and experiments," in Proc. 11th Eur. Conf. Integrated Optics, Prague, Czech Republic, 2003, pp. 277-280.

[8] R. G. Heideman and P. V. Lambeck, "Remote opto-chemical sensing with extreme sensitivity: Design, fabrication and performance of a pigtailed integrated optical phase-modulated Mach-Zehnder interferometer system," Sens. Actuators B, Chem., vol. 61, pp. 100-127, 1999.

[9] A. K. Sheridan, R. D. Harris, and P. N. Bartlett et al., "Phase interrogation of an integrated optical SPR sensor," Sens. Actuators B, Chem., vol. 97, pp. 114-121, 2004.

[10] E. Krioukov, D. J. W. Klunder, A. Driessen, J. Greve, and C. Otto, "A new type of sensor based on an integrated optical microcavity," Opt. Lett., vol. 27, pp. 512-514, 2002.

[11] S. W. Leonard et al., "Tunable two-dimensional photonic crystals using liquid-crystal infiltration," Phys. Rev. B, Condens. Matter, vol. 61, pp. R2386-R2392, 2000

[12] B. Wild et al., "Temperature tuning of the optical properties of planar photonic crystal microcavities," Appl. Phys. Lett., vol. 84, pp. 846-848, 2004

[13] H. M. H. Chong and R. M. De La Rue, "Tuning of photonic crystal waveguide microcavity by thermooptic effect," IEEE Photon. Technol. Lett., vol. 16, no. 6, pp. 1528-1530, Jun. 2004.

[14] E. A. Camargo, H. M. H. Chong, and R. M. De La Rue, "2-D photonic crystal thermo-optic switch based on AlGaAs/GaAs epitaxial structure," Opt. Exp., vol. 12, pp. 588-592, 2004.

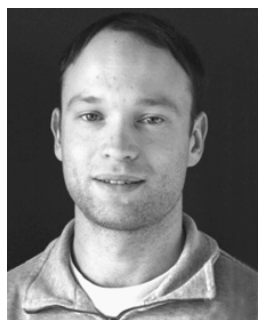

Wico C. L. Hopman (S'03) was born in Assen, The Netherlands, in 1977. He received the M.Sc. degree in electrical engineering in 2002 from the University of Twente, Enschade, The Netherlands, where he is currently working toward the Ph.D. in integrated optics.

His main focus is the development of a light induced electro-chemical etch method for silicon in order to realize photonic structures. His other research interests include photonic crystal structures, fabrication, and measurements methods.

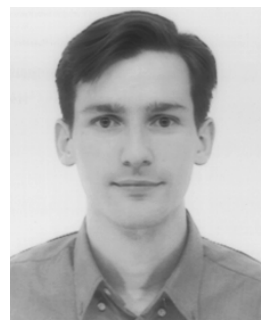

Pierre Pottier was born in Nantes, France, in 1973. He received the Ph.D. degree from the Ecole Centrale de Lyon, Lyon, France, in 2001.

$\mathrm{He}$ is currently a Research Assistant at the University of Glasgow, Glasgow, U.K. His research interests include photonic crystals and integrated optics.

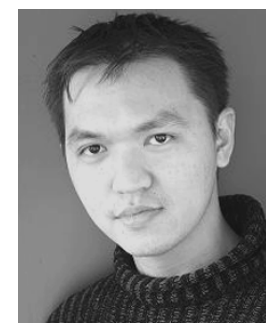

Didit Yudistira received the B.S. degree in physics from Institut Teknologi Bandung, Bandung, Indonesia, in 2001, and the M.Sc. degree in mathematical engineering, in 2003, from University of Twente, Enschede, The Netherlands, where he is currently working toward the Ph.D. degree in integrated optics.

His research interests include slow-light devices for obtaining high enhancement, numerical modeling, periodic structures, and optical waveguide theory.

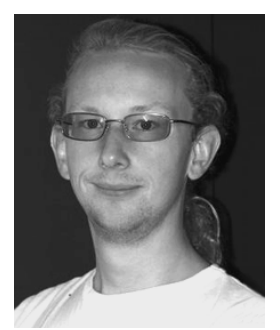

Joris van Lith was born in The Hague, The Netherlands, in 1976, He received the M.Sc. degree in applied physics in 2001 from the University of Twente, Enchade, The Netherlands, where he is currently working toward the Ph.D. degree in integrated optics.

His research interests include passive integrated optical devices for telecom and integrated optical sensors.

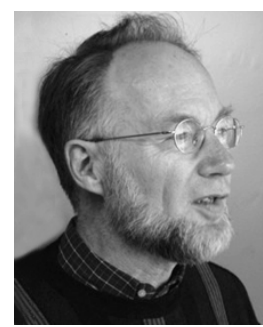

Paul V. Lambeck received the M.Sc. degree in physical chemistry from the University of Amsterdam, Amsterdam, The Netherlands, and the Ph.D. degree from the University of Twente, Enschade, The Netherlands.

He joined the University of Twente in 1964 and is currently a Full Professor in Integrated optical sensing systems, working within the Integrated Optical Micro Systems Group, MESA+ Institute. $\mathrm{He}$ is the (co-)author of about 80 papers in refereed journals.

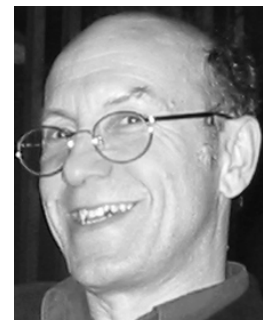

Richard M. De La Rue (M'94-SM'96-F'03) received the $\mathrm{Ph} . \mathrm{D}$. degree, which focused on closely analogous acoustic waveguides, from the University College London, London, U.K.

$\mathrm{He}$ is currently with the University of Glasgow, Glasgow, U.K., where he has been involved in guided-wave optics research for more than 30 years. He became interested in photonic crystal structures in 1993, collaborating with T. Krauss in work that led to the earliest demonstrations of photonic bandgap behavior in planar waveguide structures at optical frequencies. His research in this area has now evolved to cover compact lasers, planar micro-cavities, photonic-crystal LEDs, photonic integrated circuits (PIC's), synthetic opals, and inverse structures. More recently, his activity has extended to include photonic wire structures and, therefore, now covers all periodicity dimensions from zero to three. He has been involved in European-scale research activity through the SMILED and PICCO projects, as well as being co-leader for working group 2 (WG2) in the COST 268 Action on "Wavelength Scale Photonics." He is currently a Deputy Leader for the COST P11 Action "Linear, Nonlinear and Active Photonic Crystals." Nationally, his research has been supported by the EPSRC in the U.K. He is currently involved, in particular, in the multi-institution ultrafast photonics collaboration (UPC).

From 1999-2001, he served as an IEEE-LEOS Distinguished Lecturer. He is a Fellow of the Royal Academy of Engineering, the Royal Society of Edinburgh, and The Institution of Electrical Engineers. 


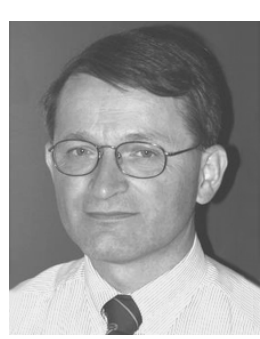

Alfred Driessen (SM'95) received the Ph.D. degree in solid-state physics from the University of Amsterdam, Amsterdam, The Netherlands, in 1982

After receiving the degree, he was a Postdoctoral Fellow researching metal hydrides at the Free University Amsterdam. In 1988, he became an Associate Professor (since 2003, he has been a Full Professor) with the Lightwave Devices Group, MESA+ Institute, University of Twente, Enschade, The Netherlands, where his research interests focused on integrated optics for optical communication. Currently, his research interests are increasingly focused on nanophotonic structures, like microresonators and photonic wires that eventually could lead to VLSI photonics.

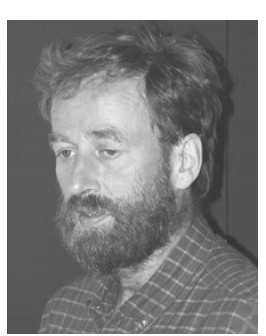

Hugo J. W. M. Hoekstra received the M.Sc. degree in experimental physics from the University of Amsterdam, Amsterdam, The Netherlands, in 1977, and the Ph.D. degree on magneto-optical effects on solids from the University of Groningen, Groningen, The Netherlands, in 1984.

Prior to receiving the Ph.D. degree, he was a high school teacher. From 1984 to 1988 , he was a Postdoctoral Fellow in the field of surfaced science at both the University of Nijmegen, Nijmegen, The Netherlands, and the University of Groningen. In 1988, he joined the Integrated Optics Microsystems Group, MESA+ Research Institute, University of Twente, Enschade, The Netherlands. His current research interests include optical waveguide theory and integrated optical sensors.

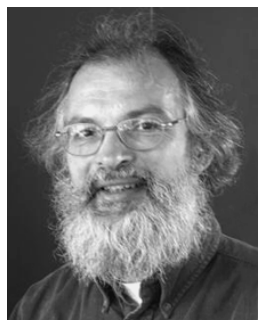

René M. de Ridder (M'94) was born April 26, 1950, in Amsterdam, The Netherlands. He received the M.S. and Ph.D. degrees in electrical engineering from the University of Twente, Enschede, The Netherlands, in 1978 and 1988, respectively. His thesis subject was on sensitive thin-film magnetic-field sensors.

Since 1981, he has been a Lecturer and Research Scientist with the University of Twente, where he is currently a member of the Integrated Optical MicroSystems Group in the MESA+ Research Institute. In 1988, his research focus turned to integrated optical devices for optical communication. A basis for his work in this field was laid during a half year of sabbatical leave at AT\&T Bell Labs, Holmdel, NJ, in 1989. His current research interests include photonic crystal structures and wavelength-selective devices. 